\title{
L'importance des échantillons corses dans les herbiers genevois
}

\author{
Daniel Jeanmonod
}

\begin{abstract}
JEANMONOD, D. (2017). Importance of Corsican specimens in the Geneva herbaria. Candollea 72: 133-142. In French, English and French abstracts. http://dx.doi.org/10.15553/c2017v721a9

The Geneva herbaria contain about 100,000 specimens collected in Corsica, resulting in the interest of the Geneva botanists, e.g. John Briquet (1870-1931) for this insular mountain. $40 \%$ of the collections are represented in the institutional database, with 331 collectors, the most frequent being notably R. Deschâtres, J. Gamisans, and R. de Litardière. According to the database, the collection dates range from 1803 to 2015 with four major peaks during the $20^{\text {th }}$ century. Ca. 250 types specimens are represented in Geneva. The geographical areas of the island are covered in a fairly homogeneous way, whereas the altitudinal belts from 0 to $100 \mathrm{~m}$ have the maximum coverage. All families and $95.7 \%$ of specific and infraspecific taxa of native flora are present in the Geneva Corsican collections. Only 197 taxa of the wild flora of the island are absent, corresponding mainly to rare, very rare or extinct taxa. These are mostly xenophytes, generally subspontaneous. These herbaria contributed a paramount wealth of information to the knowledge of Corsican botany scattered in nine research fields: flora, taxonomy, cartography, historical data, recent plant invasions, future invasions scenarios, molecular systematics and population genetics, Red List, and high biodiversity areas.
\end{abstract}

\section{Résumé}

JEANMONOD, D. (2017). L'importance des échantillons corses dans les herbiers genevois. Candollea 72: 133-142. En français, résumés anglais et français. http://dx.doi.org/10.15553/c2017v721a9

Les herbiers de Genève renferment env. 100000 échantillons récoltés en Corse, résultat de l'intérêt des botanistes genevois, dont John Briquet (1870-1931) pour cette montagne insulaire. 40\% de ces échantillons sont représentés dans la base de données institutionnelle, avec 331 collecteurs, les plus représentés étant notamment R. Deschâtres, J. Gamisans et R. de Litardière. Selon la base de données, les récoltes s'étalent entre 1803 et 2015 avec quatre pics importants durant le $20^{\mathrm{e}}$ siècle. Env. 250 specimens types de taxa corses y sont représentés. Les secteurs géographiques de l'île y apparaissent de manière assez homogène, la tranche altitudinale 0-100 m étant la plus représentée. Toutes les familles et 95,7\% des taxons spécifiques et infraspécifiques de la flore indigène sont présents dans les collections corses des herbiers de Genève. Seuls 197 taxons de la flore sauvage de l'île sont absents, en majorité des taxons rares, très rares ou disparus. Ce sont principalement des xénophytes, surtout subspontanés. Ces herbiers ont apporté une information essentielle à la connaissance de la botanique corse dans neuf champs de recherche: flore, taxonomie, cartographie, données historiques, peuplement végétal récent, évolution de la flore et scénarios futurs, systématique moléculaire et génétique des populations, Listes Rouges et zones de haute biodiversité.

\section{Key-words}

Flora - Collectors - Specimens - Altitudinal distribution - Taxonomy - Corsica

Adresse de l'auteur:

Conservatoire et Jardin botaniques de la Ville de Genève, C. P. 60, 1292 Chambésy, Suisse. E-mail: daniel.jeanmonod@ville-ge.ch 


\section{Petite histoire de la botanique en Corse}

\section{Les explorations initiales}

Les premières mentions de la flore corse semblent remonter à 1697 avec P. Boccone et son ouvrage Museo di Piante rare della Sicilia, Malta, Corsica, Italia, Piemonte Germania (Gamisans, 2009). Il faut ensuite citer C. Allioni en 1761 avec son Felicis Valle taurinensis florula corsicae. Toutefois c'est au début du $19^{\mathrm{e}}$ que des plantes corses sont vraiment répertoriées et décrites avec la Flora Gallica (1806-1807) de J.-L. LoiseleurDeslongchamps. Mais le premier botaniste à établir spécifiquement une liste de plantes corses est un botaniste allemand, $\mathrm{Ph}$. Salzmann, suite à un voyage effectué en Corse en 1820. Par la suite, dans les années 1820, l'Ile de Beauté est parcourue par des botanistes ou naturalistes italiens comme D. Viviani et G.B. Balbis, mais aussi et surtout par des français comme E. Requien, F. Robiquet et P.C.M. de Pouzolz. Peu après, un suisse de Zürich, U.A. von Salis-Marschlins, rédige un premier recensement, Aufzäblung der in Korsika (18331834), après avoir passé 3 ans sur l'Ile de Beauté comme juge attaché à un régiment de gardes suisses en Corse. Dans ces années, E. Requien jouera également un rôle de premier plan en explorant l'île et en décrivant plusieurs nouvelles espèces. Il fait, en effet, un premier voyage en Corse en 1820 puis s'y établit de 1847 jusqu'à sa mort en 1851 (DAyrat, 2003). I1 faut ensuite attendre L.J.A. Marsilly de Commines pour voir paraître en 1872 une première synthèse des connaissances de la flore corse avec le Catalogue des plantes vasculaires indigènes ou généralement cultivées en Corse, contenant 1625 espèces. Les observations et récoltes de plusieurs collecteurs sont prises en compte notamment celles de E. Revelière et P. Mabille.

Le $19^{\text {e }}$ voit passer nombre de botanistes sur l'île, des Français pour la plupart et qui y reviennent parfois plusieurs fois. On peut citer C. Burnouf, H. Coste, N. Doumet-Adanson, J. Foucaud, M. Gandoger, X.A. Gillot, A. Jordan, J.-L. Kralik, C.H.B. Moquin-Tandon, N. Roux, G. Rouy, E. Simon, l'abbé Soulié, etc. Certains d'entre eux publient leurs compte-rendu d'excursions ou leurs découvertes majeures. Il faut également citer trois collecteurs importants: le capitaine J.-F. Soleirol qui vendait ses récoltes à Loiseleur-Deslonchamps, E. Reverchon qui faisait largement commerce de ses récoltes et le $\mathrm{D}^{\mathrm{r}}$ Serafini qui était le correspondant de Viviani. Cet intérêt pour la Corse se marque par ailleurs par une session extraordinaire de la Société botanique de France en Corse en 1877, puis en 1901. Des exsiccata de presque tous ces collecteurs historiques sont présents dans nos herbiers comme le montre l'annexe 1.

\section{Le Prodrome de la Flore corse}

Selon Gamisans (2009), c'est à l'orée du 20e siècle, avec John Briquet (1870-1931) que commence l'ère moderne de la botanique corse. Briquet était directeur des Conservatoire et Jardin botaniques de Genève (CJBG) lorsqu'il fait un voyage en Corse en 1900 (Fig. 1). Comme en 1847 pour E. Burnat, cette expédition lui fait comprendre tout l'intérêt de cette île et l'incite à produire une flore de celle-ci. Sous les auspices de Burnat (et parfois avec lui), il fait 7 autres expéditions (en 1904, 1906, 1907, 1908, 1910, 1911 et 1913) et lance le Prodrome de la Flore Corse [Prodrome] (Briquet, 1910-1913). Cet ouvrage, loin d'être achevé à sa mort, est poursuivi par R. V. de Litardière qui en publie les volumes $2 / 1,3 / 1$ et $3 / 3$ (LitARDière, 1936-1955), tout en continuant à explorer la Corse (au moins 25 expéditions de 1902 à 1956). Dans cette première moitié du $20^{\mathrm{e}}$ siècle, bien d'autres botanistes explorent à plusieurs reprises la Corse comme P. Aellen, C. d'Alleizette, J. Brugère, L. Charrel, J. Chevalier, P. Cousturier, H. Gysperger, P. Le Brun, J. Lauranceau, G. Malcuit, G. Marchioni, J. Rodié, etc.

A cette époque, il faut également citer M. Conrad qui fit le lien entre bien des botanistes tant lors de la première que de la seconde moitié du 20e siècle (Bosc, 1992). Elle parcourut la Corse en tous sens, récoltant peu mais publiant ses observations et les communiquant aux nombreux botanistes qui passaient chez elle. Elle fut l'initiatrice de la conservation des plantes en Corse qu'elle défendit avec ardeur. La seconde moitié du 20 siècle est marquée par J. Gamisans . Explorant les montagnes de Corse presque chaque année dès 1966, il devient le maître incontesté de la flore corse avec de très nombreuses publications en floristique et en végétation (JEANMONOD, 2016). Il lèguera à sa mort en 2015 un herbier très important aux CJBG. En 1985, G. Bocquet, directeur des CJBG, décide de relancer le Prodrome sous forme des Compléments au Prodrome de la Flore Corse [Compléments] (Jeanmonod et al., 1987-2015). Un Comité international de la Flore Corse est créé en 1986 avec Gamisans comme président et $\mathrm{D}$. Jeanmonod comme secrétaire général. Il réunit de grands explorateurs de l'île comme G. Bosc, R. Deschâtres, G. Dutartre, J. Lambinon, M.-A. Thiébaud. Ce comité va engendrer une forte émulation avec l'organisation de nombreuses explorations, la publication de plusieurs découvertes dans les Notes et contributions à la flore de Corse, la publication des Compléments et enfin la parution d'une Flora Corsica (Jeanmonod \& Gamisans, 2007, 2013) avec de nombreuses analyses. Dès 1987, G. Paradis étudie la végétation du littoral corse avec ses étudiants (C. Piazza, C. Lorenzoni et M.-L. Pozzo di Borgo). Une prise de conscience de l'importance de la protection et de la conservation de la flore de l'île apparaît avec les travaux et les interventions de l'Agence pour le Gestion d'Espaces Naturels de Corse (AGENC) (M. Murraciole et I. Guyot), $\mathrm{du}$ Conservatoire botanique national de Porquerolles, puis du Conservatoire botanique national de Corse (CBNC) créé en 2008 (avec notamment L. Hugot, P. Spinosi, C. Panaiotis et A. Delage). Bien d'autres botanistes de toutes contrées viennent parcourir l'île, comme P. Auquier, J. Corcelle, P. Jauzein, J.-M. Tison, attirés par la beauté et l'originalité de la flore ou souvent à la recherche de taxons particuliers dans le cas d'études systématiques. 




Fig. 1. - Briquet, Burnat et Cavillier lors de leur première expédition commune en Corse [Bibliothèque des Conservatoire et Jardin botanique de la Ville de Genève]. 
De manière générale, les botanistes, amateurs ou récolteurs qui ont herborisé en Corse depuis deux siècles se comptent par centaines, que ceux-ci soient liés à une seule expédition ou à une seule région d'exploration, ou qu'ils soient revenus plusieurs fois dans l'Ile de Beauté. Certains s'y sont même établis plusieurs années de suite. On peut estimer aujourd'hui que le territoire corse a été très profondément exploré, en particulier sur ces 30 dernières années, de sa partie littorale à ses plus hauts sommets. Les explorations récentes ont toutefois montré qu'il reste encore de nombreuses nouvelles espèces à découvrir sur l'île, même si ces dernières sont souvent introduites. Il existe probablement aussi des espèces indigènes, peut-être nouvelles pour la science, qu'il reste à découvrir.

\section{La collection corse aux CJBG}

\section{Importance et structure de la collection}

De nombreuses récoltes de ces divers botanistes sont présentes aujourd'hui aux Conservatoire et jardin botaniques de Genève dans son herbier général $(G)$. On trouve également des collections plus ou moins importantes dans au moins deux des quatre herbiers fermés des CJBG, l'herbier Candolle (G-DC) et l'herbier Burnat (G-BU). En effet l'herbier G-DC renferme une quantité probablement modeste d'échantillons de Corse dont le nombre exact de spécimens ne nous est pas connu. L'herbier G-BU est plus important. Il contient environ 8000 exsiccata de Corse avec notamment les récoltes de Burnat et surtout celles de Briquet (Jeanmonod \& Charpin, 2017). On y trouve donc la plupart des spécimens cités dans le Prodrome et notamment les types décrits de Corse par Briquet.

\section{Les plantes corses de l'herbier général (G)}

L'ensemble des plantes corses de l'herbier général est estimé à environ 90000 spécimens. Tous les échantillons de Corse ont été mis dans des fourres à étiquette bleu ciel, bien distinctes de celles récoltées en Grèce (en brun), à Madagascar (en vert clair), au Paraguay (en violet), en Suisse (en vert foncé) et dans le reste du monde (en blanc). Les échantillons types sont quant à eux mis en évidence avec des étiquettes rouges. Comme tous les spécimens ont été récemment triés, on estime qu'il ne reste guère de plantes corses «cachées» dans les fourres à étiquettes blanches.

Les collections originales corses présentes dans cet herbier sont notamment celles de Aellen (env. 6500 échantillons), de Deschâtres (env. 15 000), de Gamisans (env. 12000), de Jeanmonod (env. 6000), de Litardière (env. 8000 ) et de Thiébaud (env. 4500), mais aussi celles de quelques collecteurs secondaires (pour la Corse) comme A. Charpin, J. Corcelle et M. Dittrich. On y trouve aussi bien entendu des doubles de nombreuses autres collections comme on le verra plus loin dans l'analyse des collecteurs.

\section{Les plantes corses de l'herbier Candolle (G-DC)}

Les plantes corses, disséminées dans l'herbier G-DC, sont estimées à un demi-millier. Pour les 247 spécimens déjà enregistrés dans la base de données des CJBG, on trouve une vingtaine de collecteurs (A. Bernard, Requien, Salzmann, Soleirol, P. Thomas ou encore Salis-Marschlins et Viviani) et 11 échantillons types. Les auteurs de ces derniers sont principalement de Candolle et d'Otth (par ex. Ruta corsica DC., Ligusticum cynapiifolium DC., Silene pauciflora Otth, S. requienii Otth, $S$. salzmannii Otth).

\section{Les plantes corses de l'herbier Burnat ( $G-B U$ )}

Les plantes corses de cet herbier sont présentes dans l'herbier général européen de Burnat. Elles sont aisément accessibles puisque, pour chaque taxon, elles sont clairement séparées de celles d'autres régions dans des fourres portant le numéro 8, situées juste après celles pour l'Italie (JEANMONOD \& CHARPIN, 2017). On y trouve toutes les collections du premier voyage de Burnat en 1847, mais aussi toutes celles des expéditions de Briquet faites sous les auspices de Burnat (avec ou sans sa présence), soit 5249 échantillons en tout (BRIQUET \& CAVILliER, 1922). On y découvre aussi des doubles d'au moins 51 autres collecteurs (environ 3000 échantillons en plus des récoltes de Burnat, Briquet et collaborateurs.) dont les plus importants (avec plus d'une centaine d'échantillons) sont dans l'ordre Reverchon, Mabille, Brugère, O. Debeaux et Stefani. Cet herbier renferme environ 80 échantillons types de Corse (étude en cours).

\section{Les échantillons corses dans la base de données des CJBG}

En 2016, 38998 échantillons de Corse des collections genevoises étaient entrés dans la partie corse de la base de données institutionnelle (BdD Corse). Nous estimons que ces enregistrements représentent environ 40\% des échantillons corses présents dans nos herbiers. Du fait de ce haut pourcentage, on peut estimer que cette base de données est bien représentative de l'ensemble de la collection corse. Toutefois il faut spécifier que les enregistrements effectués n'ont pas été faits au hasard mais relèvent de choix clairement définis s'articulant autour de quatre points. Ces enregistrements concernent ainsi: (1) tous les échantillons appartenant aux 12 familles analysées dans les Compléments (Asteraceae, Campanulaceae, Caprifoliaceae, Cucurbitaceae, Dipsacaceae, Globulariaceae, Lentibulariacae, Orobanchaceae, Plantaginaceae, Rubiaceae, Scrophulariaceae et Valerianaceae), ce qui représente $17,9 \%$ des taxons de la flore totale; (2) tous les échantillons récoltés par les membres des CJBG depuis 1979 (par exemple M. Dittrich, Jeanmonod, Thiébaud) et presque toutes les récoltes de Gamisans, cet ensemble de récoltes quasi exhaustives correspond à 52,8\% des données corses de la base de données; (3) au moins trois échantillons de tous les taxons non rares de Corse; (4) tous les échantillons de l'herbier général $\mathrm{G}$ de taxons considérés comme disparus, très rares, voire rares (connus d'au maximum 10 localités), ce qui représente $43 \%$ des 
taxons de la flore. En tenant compte du léger biais issus de ces choix, nous pouvons avoir une bonne idée de la richesse et de la diversité des collections corses de nos herbiers par l'analyse des échantillons enregistrés dans la BdD Corse.

\section{Composition générale des collecteurs}

Sur l'ensemble des collections, on trouve quelques 331 collecteurs cités dans la base de données, certains ayant simplement accompagnés le collecteur principal (au nombre de 274, annexe 1). Le nombre d'échantillons par collecteur est évidemment très variable avec parfois un seul échantillon pour certains collecteurs, alors que pour d'autres plus d'un millier sont probablement présents dans nos herbiers. Les collecteurs avec une estimation supérieure à 1000 échantillons sont dans l'ordre décroissant: Deschâtres (env. 15000 ), Gamisans et coll. (env. 12 000), Litardière (env. 8000), Aellen (env. 6500), Jeanmonod et coll. (5 666), Briquet-Burnat et coll. (5 249), Thiébaud et coll. (4 486), Lambinon et coll. (env. 3 000) et Reverchon (env. 1500). Ces neuf collecteurs (ou groupes de collecteurs) représentent, à eux seuls, plus de $60 \%$ des échantillons corses présents dans l'herbier. Parmi les autres collecteurs, certains sont importants historiquement, soit parce qu'ils ont décrit de nouvelles espèces ou taxons, soit parce qu'ils ont trouvé de nouvelles espèces pour l'île, soit pour d'autres raisons. On peut citer les noms de J. Aylies, Cousturier, Kralik, Mabille, Malcuit, Marchioni, Requien, Salis-Marschlins ou encore Stefani qui apparaissent chacun sur plus d'une centaine d'échantillons de Corse.

Parmi les collecteurs, on trouve 27 membres des CJBG. On y trouve aussi bien des directeurs (Briquet et Bocquet) que des conservateurs (Jeanmonod, Thiébaud, Dittrich, Charpin, etc.), des assistants scientifiques, des employés d'herbier, des jardiniers ainsi que des étudiants en master, en doctorat, voire en stage. Ils ont à eux tous récolté quelques 15000 spécimens. Ils ont été actifs essentiellement sur deux périodes, la première autour de Briquet entre 1900 et 1913, la seconde initiée par Bocquet de 1979 à nos jours.

\section{Dates et mois de récoltes}

Les échantillons les plus anciens récoltés en Corse datent de 1803 et les plus récents de 2015, ce qui représente 212 ans de récoltes. Selon notre base de données, une analyse du nombre d'échantillons récoltés année après année (Fig. 2) montre une nette progression des récoltes entre le 19e siècle (avec 1957 échantillons) et le $20^{\mathrm{e}}$ siècle (avec 34562 échantillons), cela même si cette différence est accentuée par le fait que ce sont davantage des spécimens récents qui ont été enregistrés. De fait, quatre périodes montrent des pics importants de récoltes : la période 1904-1914 (surtout récoltes de Briquet), celle de 19271936 (surtout Litardière et Aellen), de 1966-1974 (surtout Deschâtres et Gamisans) et enfin celle de 1982-1994 (surtout Gamisans, Jeanmonod et Thiébaud) avec un pic inégalé en 1986.
Durant l'année la période de récolte des échantillons s'étend sur l'ensemble des mois (janvier à décembre, Fig. 3). Elle présente une courbe plus ou moins attendue, en lien avec la période de floraison. Le maximum apparaît en mai avec 11065 récoltes, alors que le mois présentant le maximum d'espèces en fleur est celui de juin selon JEANMONOD et al. (2011).

\section{Echantillons-types}

Les collections genevoises sont réputées pour leur richesse en échantillons types. Il n'est donc pas surprenant d'en trouver dans les échantillons en provenance de Corse. Nous avons pu en localiser plus de 250 mais il en existe certainement d'autres. Outre les 11 échantillons types au moins présents dans l'herbier G-DC (voir supra), il faut en relever plus de 80 dans l'herbier Burnat (G-BU), en grande partie décrits par Briquet (par ex. Scleranthus burnatii Briq., Sedum burnatii Briq., Aquilegia litardierei Briq.), la majorité étant cependant des taxons infraspécifiques. Les autres (plus de 160) se trouvent dans l'herbier général. Ils proviennent essentiellement des collections de Litardière (135 échantillons selon JEANMONOD (2010)), de Gamisans (26 avec par ex. Seseli djianeae Gamisans, Adenostyles briquetii Gamisans, Ranunculus elisae Gamisans et $R$. sylviae Gamisans, Senecio rosinae Gamisans, Trisetum conradieae Gamisans, etc.), de Jeanmonod (Senecio serpentinicola Jeanm., Orobanche cyrnea Jeanm. et al., etc.) mais aussi d'autres collecteurs (Bellis bernardii Boiss. \& Reuter, Allium corsicum Jauzein et al., Trisetum corsicum Rouy, etc.).

\section{Répartition géographique et altitudinale des échantillons}

Selon la base de données (env. $40 \%$ des échantillons), la répartition des récoltes dans les divers secteurs de l'île (Fig. 4) est assez homogène avec néanmoins deux secteurs privilégiés : les massifs du Cintu et du Rotondu, les deux massifs les plus élevés de Corse. Ils sont suivis du secteur d'Incudine-Bavella, bien moins élevé mais particulièrement intéressant. Il faut noter également le nombre élevé de récolte dans le secteur de Bonifaciu, un tout petit secteur en surface mais particulièrement original en Corse par sa nature géologique entièrement calcaire. La répartition altitudinale (Fig. 5) présente un pourcentage de récoltes nettement plus important sur le littoral ou dans les zones proches puisque la base de données indique 9070 échantillons (36,8\% ! du total) pour l'altitude 0-99 $\mathrm{m}$. Cela est principalement dû à l'effort particulier de récolte de Thiébaud sur le littoral, sachant que toutes ses récoltes ont été enregistrées. Au-delà de ces $100 \mathrm{~m}$ d'altitude, la courbe du nombre de récoltes descend puis reste assez homogène entre 300 et $1500 \mathrm{~m}$ (avec un pic sur la tranche 800-899 m) puis descend régulièrement lorsque l'on monte en altitude. Les récoltes au-delà de $2200 \mathrm{~m}$ se comptent entre 150 et 200 échantillons par tranche de $100 \mathrm{~m}$, pour chuter à 51 pour la dernière tranche entre 2600 et $2710 \mathrm{~m}$. 


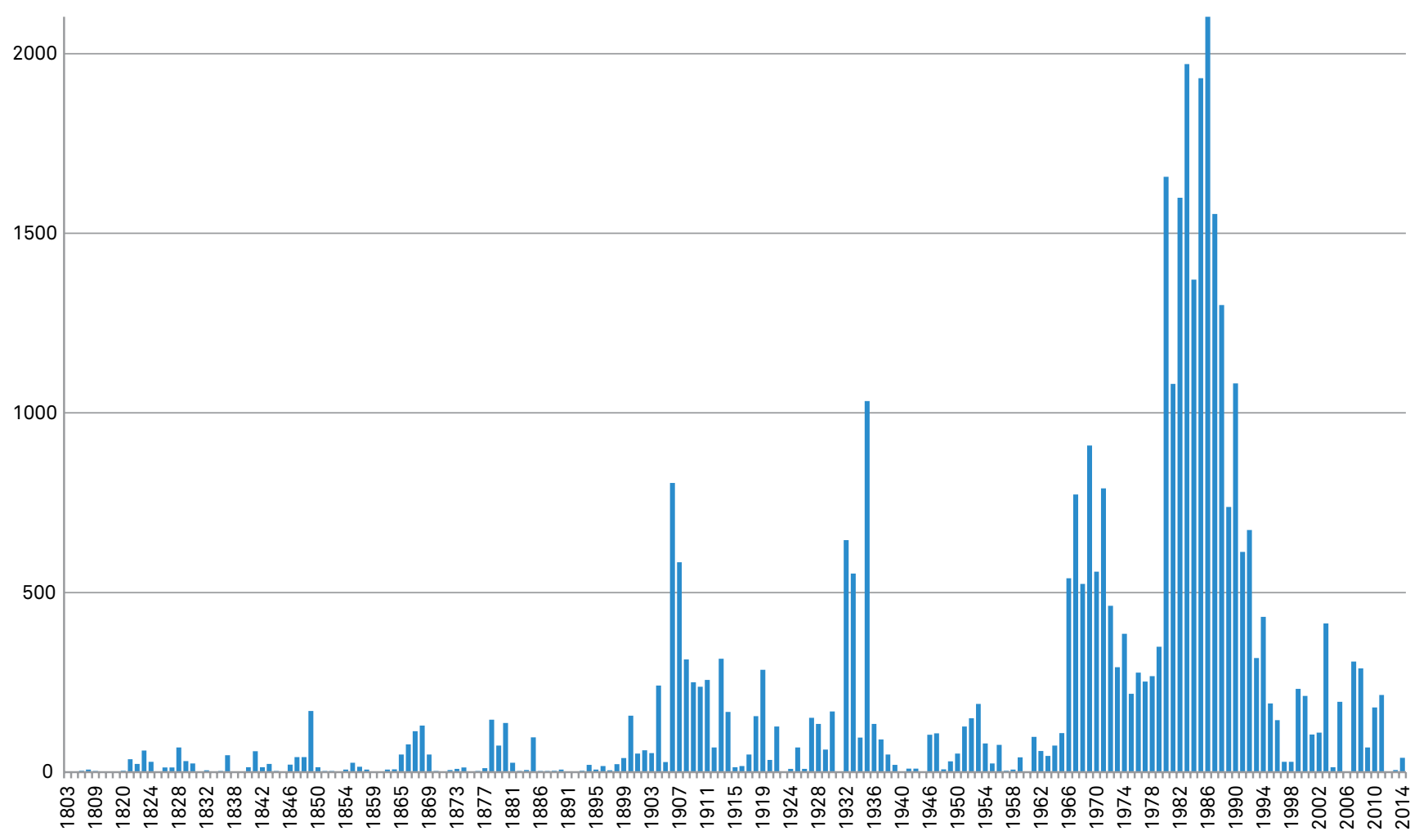

Fig. 2. - Nombre d'échantillons d'herbier par année (1803-2014) selon la base de données.

\section{Représentativité taxonomique}

Par rapport à la liste des taxons de la flore sauvage actuellement connue de Corse (Jeanmonod \& Gamisans, 2013), celle des taxons présents dans nos collections est particulièrement importante : 98,7\% des familles, 95,5\% des genres et 92,7\% des taxons spécifiques et infraspécifiques sont représentés par au moins un échantillon. Si l'on ne considère que la flore indigène, on atteint les chiffres de $100 \%$ pour les familles et $95,7 \%$ pour les taxons spécifiques et infraspécifiques. On constate que la flore corse est quasi exhaustivement présente dans les herbiers genevois avec un nombre moyen d'échantillon par taxon de 37 !

Mais plutôt que d'analyser ce qui est présent, intéressonsnous plutôt à ce qui nest pas présent dans l'herbier et pourquoi. Il s'agit de 198 taxons dont deux familles monospécifiques en Corse (Punicaceae et Pontederiaceae xénophytiques) et 38 genres presque tous monospécifiques en Corse (Agrostemma L., Alcea L., Asclepias L., Carum L., Chaetonychia Sweet, Cheirolophus Cass., Cicer L., Convallaria L., Corallorbiza Gagnebin, Coriandrum L., Cotoneaster Medik., Cydonia Mill., Diphasiastrum Holub, Dracunculus Mill., Eichhornia Kunth, Eriobotrya Lindl., Gaillardia Foug., Geropogon L.,
Hermodactylus Mill., Hesperis L., Hibiscus L., Hyacinthus L., Kernera Medik., Lagenaria Ser., Levisticum Hill, Mespilus L., Ocimum L., Phaseolus L., Punica L., Secale L., Spinacia L., Thapsia L., Tristagma Poepp., Triticum L. (avec 2 spp.), Tulipa L. (avec 2 spp.), Turgenia Hoffm., Vaccaria Wolf et Zea L.). De fait ces taxons manquants sont pour plus de la moitié (51\%) des xénophytes (101) et très rarement des endémiques (12). Comme on peut s'y attendre ce sont en majeure partie des taxons rares ou très rares (151), voire considérés comme disparus de Corse (42). Seuls cinq taxons considérés comme localisés ou peu fréquents ne sont pas représentés dans l'herbier.

L'absence de l'ensemble de ces taxons dans nos herbiers peut être interprétée de quatre façons:

- ce sont des taxons vus par des auteurs du $19^{\mathrm{e}}$ et qui ont disparu depuis (32) (par ex. Avena strigosa Schreb., Crocus sativus L., Cuscuta epilinum Weihe, Hyacinthus orientalis L., Linum usitatissimum L., Phelipanche ramosa (L.) Pomel, Triticum turgidum L., Tulipa agenensis DC. et T. raddii Reboul, Vaccaria hispanica (Mill.) Rauschert) notamment du fait de l'abandon de certaines cultures. Certaines de ces 


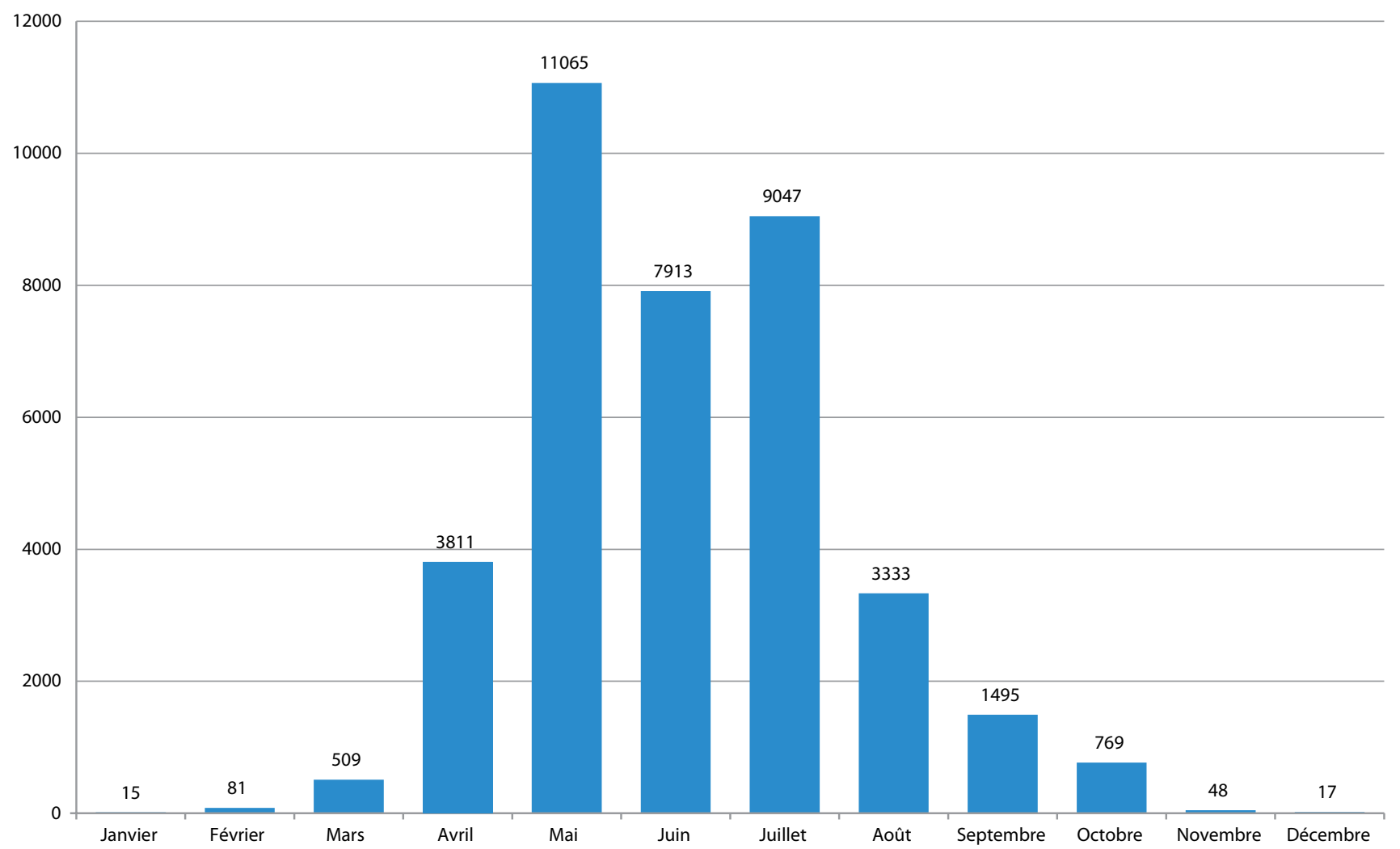

Fig. 3. - Nombre d'échantillons d'herbier collectés par mois selon la base de données.

anciennes mentions pourraient d'ailleurs être des erreurs. Mais sans les échantillons à disposition, il est impossible de se prononcer;

- ce sont en grande partie des taxons subspontanés (65), issus de cultures plus ou moins banales, comme Brassica oleracea L., Helianthus annuus L., Lactuca sativa L., Phaseolus vulgaris L. ou Vicia faba L. Ces plantes sont dans la Flora Corsica car elles ont été signalées comme "parfois subspontanées» mais on n'a, semble-t-il, jamais pris la peine de les récolter. Dans certains cas, leur subspontanéité serait d'ailleurs à vérifier;

- ce sont des taxons adventices (28) qui ont été très rarement récoltés et dont nous ne possédons aucun spécimen. Certains, de mention très ancienne, seraient d'ailleurs à vérifier dans d'autres herbiers où ils pourraient se trouver;

- ce sont des taxons endémiques (12), très souvent issus de révisions récentes et qui nont peut-être pas été distinguées au sein de nos échantillons. Nous pensons notamment au genre Ophrys L. qui a explosé ces dernières années, de même qu'aux genres Colchicum L., Epipactis Zinn, Limonium Mill. et Orchis L. Une révision de nos échantillons pourrait révéler une partie au moins de ces taxons manquant: Colchicum arenasii Fridl., Limonium greuteri Erben, Ophrys aprilia Devillers \&
Devillers-Tersch., O. eleonorae Devillers-Tersch. \& Devillers, O. marmorata G. Foelsche \&W. Foelsche, O. panormitana subsp. praecox (Corrias) Paulus \& Gack, O. peraiolae G. Foelsche et al., O. zonata Devillers-Tersch. \& Devillers, Orchis corsica Viv. et O. ichnusae (Corrias) Devillers-Tersch.\& Devillers. On peut encore mentionner Cyclamen balearicum Willk. et Verbascum conocarpum Moris subsp. conocarpum.

Apport de l'herbier et de ses spécimens à la connaissance de la flore Corse

L'exhaustivité et la représentativité de nos herbiers ont permis des apports importants à la connaissance de la flore Corse avec pour résultat de nombreuses publications. On peut donc insister sur l'utilité multiple et trop souvent méconnue des herbiers, tout particulièrement des grands herbiers tels que celui de Genève. Cette utilité et cet apport ont pu se décliner, dans le cadre de la flore corse, en une dizaine de champs d'application et permettent ainsi l'établissement de:

- flores générales, comme le Prodrome ou encore la Flora Corsica avec nombre de données extraites directement des échantillons d'herbier (descriptions, dates de floraison, stations, altitude, etc.); 
- travaux monographiques consacrés à des groupes taxonomiques comme les Compléments;

- cartes de distribution des taxons soit pour l'île, soit sur une plus large échelle comme l'Atlas Florae Europeae (Kurrto et al., 2004, 2007, 2010, 2013);

- données fiables servant la vérifications de données historiques, permettant de valider la présence de certains taxons considérés comme douteux car non retrouvés sur le terrain depuis leur mention historique dans la littérature. C'est ainsi que nombre de taxons ont pu être confirmés ou, qu'au contraire, d'autres ont pu être éliminés suite à une redétermination (par exemple Carex praecox Schreb., Eleocharis quinqueflora (Hartmann) O. Schwarz, Herniaria birsuta subsp. cinerea (DC.) Arcang., Juncus acutiflorus Hoffm., J. compressus Jacq., etc.);

- travaux sur l'histoire du peuplement végétal récent de l'île permettant de dater plus précisément l'arrivée de certaines espèces grâce aux premiers échantillons récoltés. Les diverses récoltes d'un taxon permettent parfois d'en suivre la progression sur l'île, par exemple pour Cotula coronopifolia L. et Paspalum dilatatum Poiret (NATALI \& Jeanmonod, 1996) ou même de tracer la disparition de certaines espèces (par ex. le Xanthium strumarium L. remplacé par $X$. italicum Moretti (JEAnMonod, 1998);

- modèles de scénarios de l'évolution future de la flore (par l'arrivée de xénophytes), construits sur le couplement des données de l'herbier avec celles de la littérature (cf. JEANMONOD et al., 2011a, 2011b);

- travaux en systématique moléculaire et génétique des populations grâce à l'extraction d'ADN des échantillons d'herbier comme sur le genre Silene L. (Leuzinger et al., 2015 ; Naciri et al., 2017);

- «Listes Rouges» présentant le statut de conservation suivant les Catégories et les Critère de l'UICN (UICN, 2012) de chacune des espèces, comme cela a été fait récemment pour la Corse (Delage \& Hugot, 2015);

- zones de haute biodiversité servant à la protection des sites. Les nombreuses données publiées ou envoyées au Conservatoire botanique national de Corse ont permis de mieux cerner ces zones, comme par exemple le «Coscione» (Jeanmonod, 2009).

Les herbiers continuent donc aujourd'hui de représenter une source inépuisable d'informations, notamment grâce aux nouvelles technologies qui permettent une extraction et un nouveau traitement des informations mais aussi grâce à l'accès à l'ADN contenu dans les spécimens d'herbier.
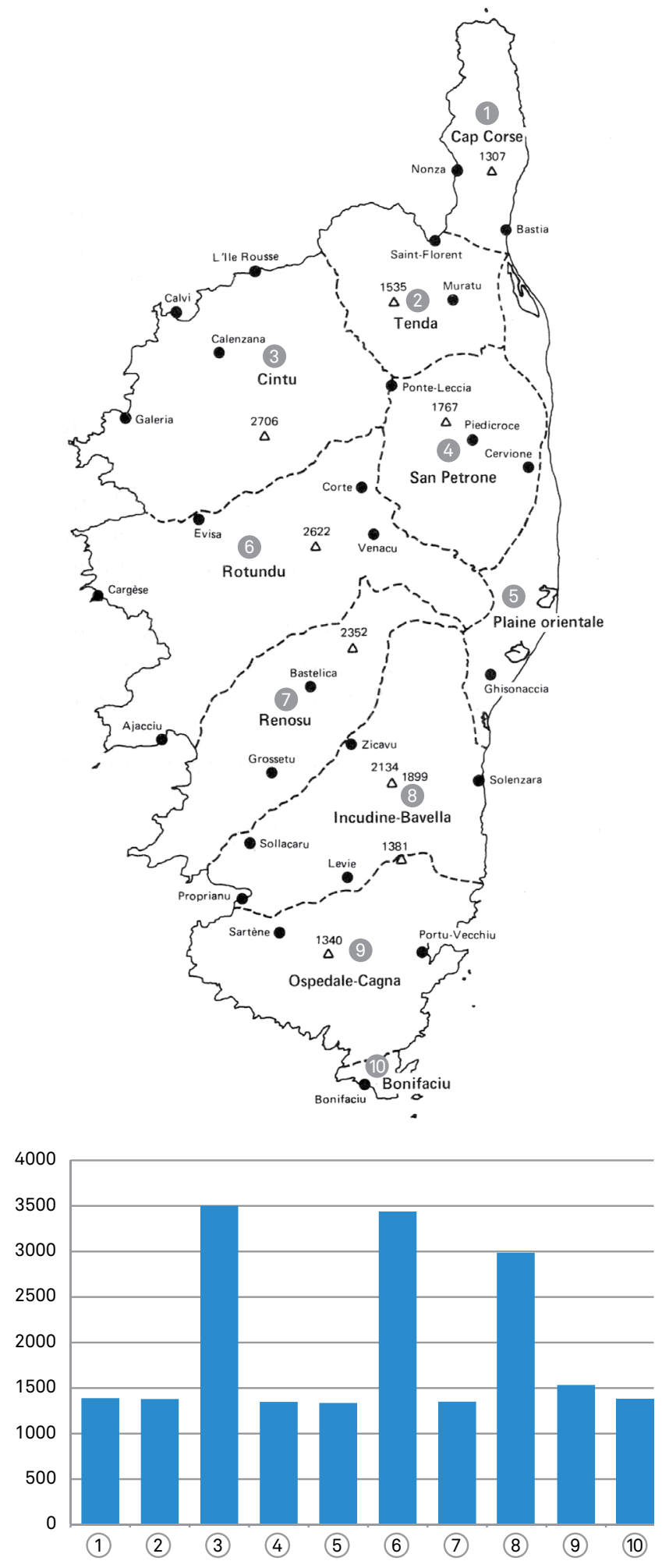

Fig. 4. - Carte de la Corse montrant les secteurs géographiques de l'île et le nombre d'échantillons d'herbier par secteur selon la base de données. 


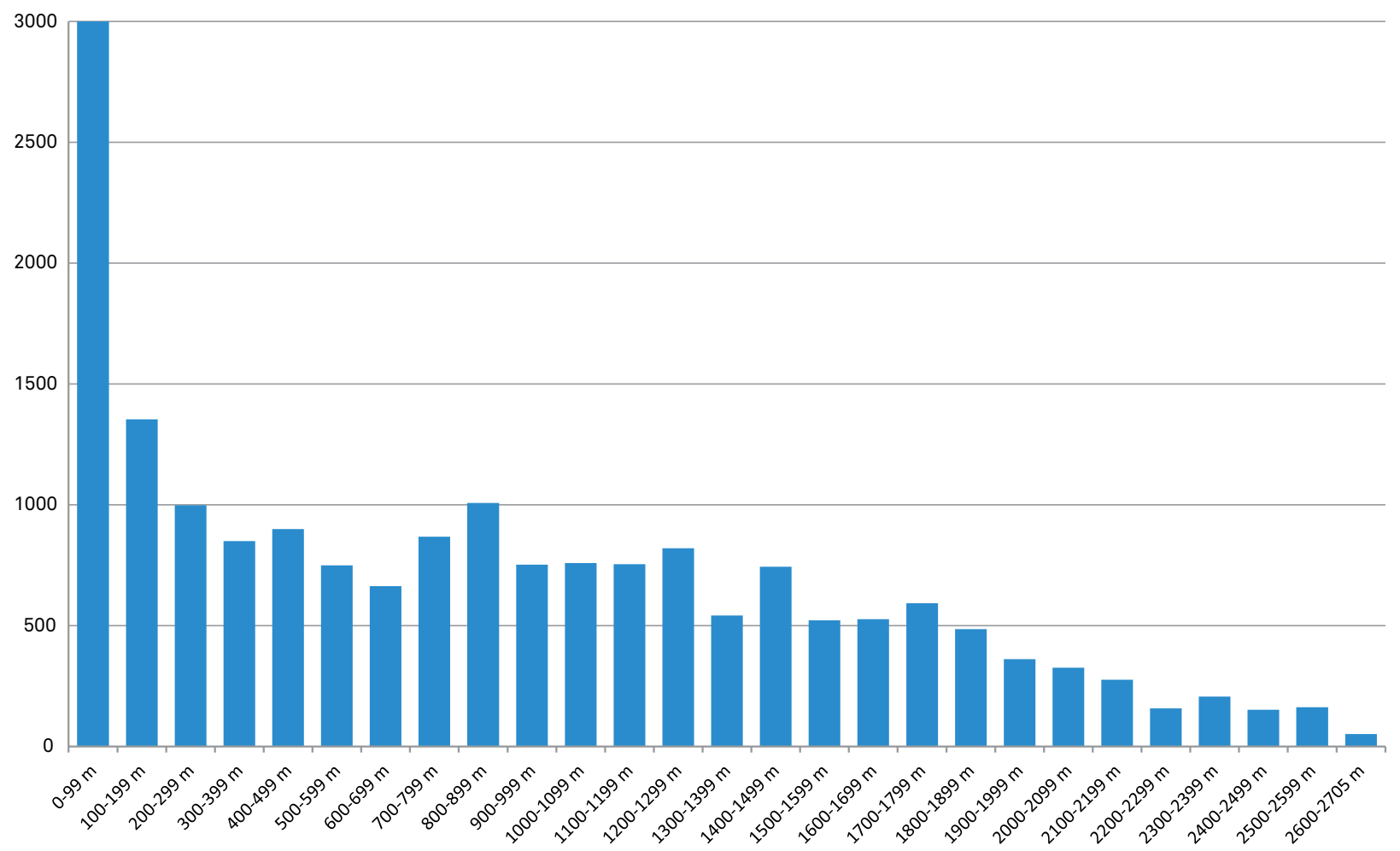

Fig. 5. - Nombre d'échantillons d'herbier selon l'altitude, par tranche de $100 \mathrm{~m}$, selon la base de données. La première tranche (9070 échantillons) a été tronquée à 3000 pour permettre une meilleure visibilité des autres tranches.

\section{Remerciements}

Je remercie Philippe Clerc pour sa relecture et les corrections apportées à ce manuscrit, Martin Cramatte pour la mise en page des graphiques, Patrick Bungener et Martin Callmander pour leurs suggestions et propositions de corrections ainsi que Laurent Gautier pour son aide dans la traduction du résumé en anglais.

\section{Références}

Bosc, G. (1992). Marcelle Conrad (1897-1990). Bull. Soc. Sci. Hist. Nat. Corse 661: 18-26.

Briquet, J. (1910-1913). Prodrome de la flore corse. Vol. 1, 2/1. Genève. Briquet, J. \& F. Cavillier (1922). Emile Burnat, autobiographie publiée avec une étude sur le botaniste et son cuvre, des souvenirs et documents divers. Conservatoire botanique, Genève.

Briquet, J. \& R. de Litardière (1936-1955). Prodrome de la flore corse Vol. 2/2, 3/1, 3/2. Paris.

Dayrat, B. (2003). Les botanistes de la flore de France. Museum national d'Histoire naturelle, Paris.

Delage, A. \& L. Hugot (2015). Liste rouge régionale de la flore vasculaire de Corse. Conservatoire botanique national de Corse, Office de l'environnement de la Corse, Corte.

Gamisans, J. (2009). Petite histoire de la botanique en Corse. Stantari $15: 24-29$.
Jeanmonod, D. (1998). Xanthium subg. Xanthium et Helichrysum italicum, deux cas taxonomiques ardus. Candollea 53 : 435-457.

Jeanmonod, D. (2009). Le Coscione, un Galapagos corse? Stantari $18: 30-35$.

Jeanmonod, D. (2010). Typification des taxons corses de l'herbier de Litardière (G). Candollea 65 : 23-44.

JEanmonod, D. (2016). Nécrologie. Jacques Gamisans (1944-2015). Candollea $71:$ 379-381.

Jeanmonod, D., G. Bocquet \& H.-M. Burdet (1987-2015). Compléments au Prodrome de la Flore Corse. Conservatoire et Jardin botaniques de la Ville de Genève.

Jeanmonod, D. \& A. Charpin (2017). L'Herbier Burnat (G-BU). Candollea $72: 143-153$.

Jeanmonod, D. \& J. Gamisans (2007). Flora Corsica. Edisud.

Jeanmonod, D. \& J. Gamisans (2013). Flora Corsica. Ed. 2. Bull. Soc. Bot. Centre-Ouest 39.

Jeanmonod, D., A. Schlüssel \& J. Gamisans (2011a). Analyse de la flore Corse: aspects biologiques. Candollea 66:5-25.

Jeanmonod, D., A. Schlüssel \& J. Gamisans (2011b). Status and trends in the alien flora of Corsica. Bull. OEPP/EPPO Bull. 41: 85-99.

Kurrto, A., S.E. Fröhner \& R. Lampinen (2007). Atlas Florae Europaeae. Vol. 14. Rosaceae (Alchemilla and Aphanes). Committee for Mapping the Flora of Europe \& Societas Biologica Fennica Vanamo. Helsinki. 
Kurrto, A., R. Lampinen \& L. Junikka (2004). Atlas Florae Europaeae. Vol. 13: Rosaceae (Spiraea to Fragaria, excl. Rubus). Committee for Mapping the Flora of Europe \& Societas Biologica Fennica Vanamo. Helsinki.

Kurtto, A., A.R. Senni kov \& R. Lampinen (2013). Atlas Florae Europaeae. Vol. 16: Rosaceae (Cydonia to Prunus, excl. Sorbus). Committee for Mapping the Flora of Europe \& Societas Biologica Fennica Vanamo. Helsinki.

Kurtto, A., H.E. Weber, R. Lampinen \& A.R. Sennikov (2010). Atlas Florae Europaeae. Vol. 15 : Rosaceae (Rubus). Committee for Mapping the Flora of Europe \& Societas Biologica Fennica Vanamo. Helsinki.

Annexe 1. - Liste des collecteurs de la flore Corse dans les herbiers genevois, par période de demi-siècle.

Dans le cas de récoltes s'étendant sur deux périodes, seule la période la plus importante est retenue; dans le cas de groupes de collecteurs, seul le premier collecteur est considéré. En gras sont indiqués les collecteurs dont les collections complètes originales corses se trouvent dans les herbiers genevois.

\section{Collecteurs entre 1800 et 1849}

Aubry de la Mottraie, ?; Balbis, G.B.; Bastard, T.; Becker, S.P.; Belanger, C.; Bernard, A.; Besson, ?; Bourdet, P.F.M.; Bourgeau, E.; Bubani, P.; Chirriaux, ?; Clarion, J.; Clement, ?; Daenen, P.; De Forestier, A.; Dufour, J.-M.L.; Durand, P.; Fauche, M.; Forestier, M.A. de; Forrest, G.; Gay, J.E.; Glastien, F.; Graves, ?; Haller, A.; Jullien, A.; Kralik, J.-L.; Labillardiere, J.J.H. de; Leo, P.; Leveille, ?; Maire, ?; Merat de Vaumartoise, J.; Noisette, L.C.; Poiteau, P.A.; Pouzolz, P.M. C.; Prost, ?; Raquin, X.; Requien, E.; Robert, G.N.; Rombure, ?; Salis-Marschlins, C.U.; Salzmann, P.; Schleicher, J.C.; Schlim, L. J.; Serafini, ?; Sieber, F.W.; Soleirol, J.F.; Teillard, ?; Theveneau, A.V.; Thibaud, E.; Thiriaux, ? ; Thomas, E.; Thomas, P.; Ventenat, E.P.; Viviani, D.

\section{0-1899}

Alioth, F.S.; André, A.; Andrée, M.; Autheman, A.; Ayasse, A.E.; Bamberger, J.G.; Bernet, H.; Bernoulli, W.; Besnes, J.P.; Boreau, A.; Boullu, A.; Burnouf, C.; Carrier, ?; Caruel, T.; Chabert, A.C.; Chevalier, E.; Chodat, R.; Correvon, H.; Debeaux, O.; Degors, A.; Duby, J. E.; Fabre,J.H.; Feer, H.; Forsyth-Major, C.I.; Foucaud, J.; Gandoger, M.; Gaudefroy, E.; Gillot, F.X.; Girod, L.-A.; Guillon, A.; Halle, A.; Hanry, H.; Hervier-Basson, J.; Huon, A.; Huvy, ?; Jacob, B.; Jahandiez, E.; Jordan, C.T.A.; Kesselmeyer, P.A.; Le Grand, A.; Legrand, G.; Levier, E.; Mabille, P.; Mandon, E.; Marsilly, L.J.A.; Martins, C.; MoquinTandon, C.H.B.; Mouillefarine, E.; Numberg, ?; Ozanon, C.; Perrier de la Bâthie, E.; Pieri, A.; Poisson, J.; Raymond, E.; Revelière, E.; Reverchon, E.; Revillon, ?; Roux, N.; Rouy, G.; Saint-Lager, J.B.; Schiffmann, A.; Stefani, J.; Tillet, P.; Trabut, L.C.; Vaucher, H.
Leuzinger M., Y. Naciri, P.-E. du Pasquier \& D. Jeanmonod (2015). Molecular diversity, phylogeography and evolutionary relationships of the Silene paradoxa group of section Siphonomorpha (Caryophyllaceae) Pl. Syst. Evol. 301 : 265-278.

Naciri, Y., P.-E. du Pasquier, M. Lundberg, D. Jeanmonod \& B. OxeLman (2017). A phylogenetic circumscription of Silene section Siphonomorpha (Caryophyllaceae) in the Mediterranean Basin. Taxon 66: 91-108.

Natali, A. \& D. Jeanmonod (1996). Flore analytique des plantes introduites de Corse. Conservatoire et Jardin botaniques de la Ville de Genève.

UICN (2012). Catégories et Critères de la Liste Rouge de l'UICN: Version 3.1. $2^{\text {nd }}$ ed. Gland et Cambridge.

\section{0-1949}

Aellen, P.; Alleizette, C. d'; Ambrosi, A.; Audigier, P.; Aylies, J.; Beauverd, G.; Behr, O.; Bicknell, C.; Briquet, J.; Brugère, J.; Burnat, E.; Camus, F. A.; Chevalier, J.; Chouard, P.; Colonna-Cesari, X.; Coste, H.; Cousturier, P.; Duffour, C.; Falley, ?, ; Fiedler, O.; Gsell, R.; Guerand, M.; Gysin, M.; Gysperger, H.; Houard, C.; Issler, E.; Jacquet, F.; Jansen, P.; Jaquet, F.; Kofler, L.; Kolas, ?; Kugler, G.; Kükenthal, G.; Lamar, P.; Le Brun, P.; Litardière, C. H.; Litardière, R.V.; Ludi, W.; Maire, R.; Malcuit, G.; Marchioni, T.; Marty, L.; Meiklejohn, R.F.; Meylan, O.; Nicoli, R.M.; Orsini, P.; Poeverlein, H.; Reynier, A.; Rikli, M.A.; Roche, P.; Rodie, J.; Ronniger, K.; Rotges, R.; Sagorski, E.A.; Saint-Yves, A.; Simon, E.; Simonet, J.; Soulié, J.A.; Spencer, M.F.; Thellung, A.; Wilczek, E.; Zschacke, H.

\section{0-2000}

Aboucaya, A.; Auquier, P.; Baguette, M.; Bellotte, W.; Bigot, A.; Bigot, J.-L.; Binder, M.; Bocquet, G.; Bonfils, J.; Bosc, G.; Campbell, M.S.; Chaffin, C.; Charpin, A.; Cirujano, S.; Clauzade, G.; Conrad, M.; Coppejans, E.; Corcelle, J.; De Santi, ?; Demoulin, V.; Deschâtres, R.; Dinter, I.; Dittrich, M.; Ducerf, G.; Duperrex, A.; Dupre, ?; Dutartre, G.; Duvigneaud, J.; Fabre, G.; Fischer, A.; Gamisans, J.; Garraud, L.; Geissler, P.; Grand, ?; Greuter, W.; Grisoni, ?; Guillot, J.; Guyot, I.; Hébrard, J.-P.; Hinz, P.-A.; Hopflinger, F.; Jallu, J.; Jauzein, P.; Jeanmonod, D.; Lambinon, J.; Lauranceau, J.; Loiseau, J.-E.; Lorenzoni, C.; Mari, M.-G.; Marzocchi, J.-F.; Mermoud, M.; Molinier, R.; Muracciole, M.; Panis, J.; Paradis, G.; Pelgrims, C.; Pioli, A.; Ponel, P.; Rasbach, H.; Reid, D.A.; Renard, R.; Retz, B. de; Rondon, Y.; Salanon, R.; Schlüssel, A.; Schumacker, R.; Seitter, H.; Simon, C.; SimonStuder, C.; Stemmler, ?; Terre, J.; Thibault, J.-C.; Thiébaud, M.-A.; Vilmorin, R. de; Vitek, E.; Vivant, J.; Vogt, R.; Weibel, R.

\section{0-2015}

Alphand, J.; Clerc, P.; Delage, A.; Delaugerre, M.; Desfayes, M.; Du Pasquier, P.-E.; Faggio, G.; Georges, N.; Marsault, L.; Michaud, H.; Nicolier, E.; Pax, N.; Rubio, P.; Scouppe, M.; Tison, J.-M. 